\title{
BOUSSINESQ - LÖVE MATHEMATICAL MODEL ON A GEOMETRICAL GRAPH
}

\author{
A. A. Zamyshlyaeva1, alzama@mail.ru \\ A. $\boldsymbol{V}$. Lut $\boldsymbol{t}^{1}$, lutti74@rambler.ru \\ ${ }^{1}$ South Ural State University, Chelyabinsk, Russian Federation.
}

\begin{abstract}
Of concern is the Boussinesq - Löve mathematical model describing longitudinal vibrations in the elements of construction that can be represented in the form of a finite connected oriented graph. Differential equations on graphs is a relatively new piece of mathematical knowledge. The article deals with Sobolev type equations on graphs. The Fourier method is used for solution of the problem. The article besides introduction, conclusion and list of references contains four paragraphs. The first paragraph describes the properties of eigenvalues and eigenfunctions of the problem on a graph. The second section is devoted to specific examples of solutions of the Sturm - Liouville problem. In the third paragraph, by applying the Fourier method, the solutions of the Boussinesq - Löve problem are found. In the last paragraph, the possibility of usage of the Fourier method for Boussinesq - Löve problem for some finite connected oriented graphs is justified.

Keywords: Sobolev type model, geometrical graph, Fourier method, Sturm - Liouville problem.
\end{abstract}

\section{Introduction}

Let $G=G(\mathfrak{D}, \mathfrak{E})$ be a finite connected oriented graph, where $\mathfrak{D}=\left\{V_{i}\right\}$ is the set of vertices, and $\mathfrak{E}=\left\{E_{i}\right\}$ is the set of edges. Each edge is characterized by two numbers $l_{j}, d_{j} \in \mathbb{R}_{+}$, denoting the length and cross-sectional area of the edge $E_{j}$ respectively. On a graph $G$, consider the Boussinesq - Löve equations [1]

$$
\begin{gathered}
\lambda u_{j t t}-u_{j x x t t}=\alpha u_{j x x} \\
\text { for all } x \in\left(0, l_{j}\right), t \in \mathbb{R} .
\end{gathered}
$$

Equations of the form (1) in $\Omega \subset \mathbb{R}^{n}$ were studied previously using the phase space method [2, 3]. Optimal control of solutions for equation (1) was studied in $[4,5]$. In [6] the phase space of the modified Boussinesq equation was constructed. In addition the algorithm of numerical modelling of Boussinesq - Löve waves was developed [7].

For equations (1) at each vertex of the graph define the conditions

$$
\begin{gathered}
\sum_{E_{j} \in E^{a}\left(V_{i}\right)} d_{j} c_{j}(0) u_{j x}(0, t)-\sum_{E_{m} \in E^{\omega}\left(V_{i}\right)} d_{m} c_{m}\left(l_{m}\right) u_{m x}\left(l_{m}, t\right)=0, \\
u_{j}(0, t)=u_{k}(0, t)=u_{m}\left(l_{m}, t\right)=u_{n}\left(l_{n}, t\right),
\end{gathered}
$$

where $E_{j}, E_{k} \in E^{\alpha}\left(V_{i}\right), E_{m}, E_{n} \in E^{\omega}\left(V_{i}\right), t \in \mathbb{R}$. Here $E^{\alpha(\omega)}\left(V_{i}\right)$ denotes the set of edges staring (ending) at the vertex $V_{i}$. Condition (2) indicates that the flow through each vertex must be equal to zero, and condition (3) indicates that the solution $u=\left(u_{1}, u_{2}, \ldots, u_{j}, \ldots\right)$ at each vertex must be continuous. In the particular case when the graph $G$ consists of one edge and two vertices, condition (3) vanishes and condition (2) becomes a Neumann 
condition. Function $u_{j}(x, t)$ defines a longitudinal displacement at point $x$ at the moment $t$ for the $j$-th element of construction.

Differential equations on graphs is a relatively new piece of mathematical knowledge. The first publications in this area appeared in the last decade of the last century, the first monograph was published in 2004. [8] and was devoted to the study of qualitative properties of differential equations on manifolds network type.

Sobolev type equations on graphs were first studied in 2002 [9]. In [10, 11] in the solution of such equations there arose a Sturm - Liouville problem

$$
a_{j}(x) u_{j}-\left(c_{j}(x) u_{j x}\right)_{x}=\lambda u_{j},
$$

with conditions (2), (3), however, in the particular case $\left(c_{j}(x) \equiv 1, a_{j}(x) \equiv a=\right.$ const), and the proposed approach had little in common with the results of [8]. Further research [11] has led to a new, more general, Sturm - Liouville problem of the form (2) - (4). This fact has motivated to consider problem $(2)-(4)$, which is a natural generalization of the previously discussed problems. Abstract results obtained in [11] are final and exhaustive. However, there disadvantage is the lack of any concrete examples and illustrations of the results. The aim of this work is to find the solution of the Boussinesq - Löve problem for some finite, connected oriented graphs.

The article besides introduction, conclusion and list of references contains four paragraphs. The first paragraph describes the properties of eigenvalues and eigenfunctions of the problem taken from [11]. The second section is devoted to specific examples of solutions of the Sturm - Liouville problem. In the third paragraph, by applying the Fourier method, the solutions of the Boussinesq - Löve problem are found. In the last paragraph, the possibility of usage of the Fourier method for Boussinesq - Löve problem for some finite connected directed graphs is vindicated.

\section{Eigenvalues and eigenfunctions of the Sturm - Liouville problem}

Consider a Hilbert space

$$
L_{2}(G)=\left\{g=\left(g_{1}, g_{2}, \ldots, g_{j}, \ldots\right), g_{j} \in L_{2}\left(0, l_{j}\right)\right\}
$$

with inner product

$$
\langle g, h\rangle=\sum_{j} d_{j} \int_{0}^{l_{j}} g_{j} h_{j} d x
$$

and a Banach space

$$
\mathfrak{U}=\left\{u=\left(u_{1}, u_{2}, \ldots, u_{j}, \ldots\right): u_{j} \in W_{2}^{1}\left(0, l_{j}\right) \text { and }(0.2) \text { holds }\right\}
$$

with norm

$$
\|u\|_{\mathfrak{U}}^{2}=\sum_{j} d_{j} \int_{0}^{l_{j}}\left(u_{j x}^{2}+u_{j}^{2}\right) d x .
$$

Definition 1. Not identically equal to zero function $u=\left(u_{1}, u_{2}, \ldots, u_{j}, \ldots\right)$ is called an eigenfunction of problem (0.2) - (0.4) for the operator

$A=\left(\frac{\partial}{\partial x}\left(-c_{1}(x) \frac{\partial}{\partial x}\right)+a_{1}(x), \frac{\partial}{\partial x}\left(-c_{2}(x) \frac{\partial}{\partial x}\right)+a_{2}(x), \ldots, \frac{\partial}{\partial x}\left(-c_{j}(j) \frac{\partial}{\partial x}\right)+a_{j}(x), \ldots\right)$, 
if there exists such a number $\lambda$ that the function $u$ is a classical solution of the following problem:

$$
\begin{gathered}
a_{j}(x) u_{j}-\left(c_{j}(x) u_{j x}\right)_{x}=\lambda u_{j}, \\
u_{j}(0, t)=u_{k}(0, t)=u_{m}\left(l_{m}, t\right)=u_{n}\left(l_{n}, t\right), E_{j}, E_{k} \in E^{a}\left(V_{i}\right) \cup E^{\omega}\left(V_{i}\right), \\
\sum_{E_{j} \in E^{a}\left(V_{i}\right)} d_{j} c_{j}(x) u_{j x}(0, t)-\sum_{E_{k} \in E^{\omega}\left(V_{i}\right)} d_{k} c_{k}(x) u_{k x}\left(l_{k}, t\right)=0 .
\end{gathered}
$$

The number $\lambda$ is called an eigenvalue (corresponding to an eigenfunction $u$ ).

It is easy to verify that an eigenfunction of problem $(2)-(4)$ for all $v \in \mathfrak{U}$ satisfies an integral identity

$$
\sum_{E_{j} \in \mathfrak{E}} d_{j} \int_{0}^{l_{j}}\left(c_{j}(x) u_{j x} v_{j x}+a_{j}(x) u_{j} v_{j}\right) d x=\lambda \sum_{E_{j} \in \mathfrak{E}} d_{j} \int_{0}^{l_{j}} u_{j} v_{j} d x .
$$

Definition 2. Nonequal to zero function $u \in \mathfrak{U}$ is called a generalized eigenfunction of problem (2) - (4) for an operator $A$ if there exists such a number $\lambda$ (an eigenvalue corresponding to an eigenfunction $u$ ) that a function $u$ for all $v \in \mathfrak{U}$ satisfies an integral identity (8).

Further we will consider only the eigenvalues and the corresponding eigenfunctions, normalized by the condition $\|u\|_{L_{2}(G)}=1$. It is convenient to consider the identity (8), which defines the generalized eigenfunctions, as the equality of the scalar products in the space $L_{2}(G)$ and in the space $U$ respectively. Let

$$
m=\min _{j}\left(\min _{x \in\left(0, l_{j}\right)} a_{j}(x)\right) .
$$

(Here we do not assume that $a_{j}(x) \geq 0$ ). Therefore, the scalar product (equivalent to standard) in $\mathfrak{U}$ can be defined by

$$
[u, v]=\sum_{E_{j} \in \mathfrak{E}} d_{j} \int_{0}^{l_{j}}\left(c_{j}(x) u_{j x} v_{j x}+\tilde{a}_{j}(x) u_{j} v_{j}\right) d x .
$$

The identity (9) can then be rewritten as

$$
[u, v]=(\lambda-m+1)\langle u, v\rangle \text {. }
$$

Theorem 1. Eigenvalues $\lambda_{1}, \lambda_{2}, \ldots$ of problem (2) - (4) for an operator

$A=\left(\frac{\partial}{\partial x}\left(-c_{1}(x) \frac{\partial}{\partial x}\right)+a_{1}(x), \frac{\partial}{\partial x}\left(-c_{2}(x) \frac{\partial}{\partial x}\right)+a_{2}(x), \ldots, \frac{\partial}{\partial x}\left(-c_{j}(j) \frac{\partial}{\partial x}\right)+a_{j}(x), \ldots\right)$ are real and $\lambda_{s} \rightarrow+\infty$ as $s \rightarrow \infty$. Eigenvalues satisfy the inequality

$$
\lambda_{s}>m=\min _{j}\left(\min _{x \in\left(0, l_{j}\right)} a_{j}(x)\right)
$$

in all cases, except $a_{j}(x)=a_{i}(x)=$ const for all $i, j$. 
If $a_{j}(x)=a_{i}(x)=$ const for all $i, j$, then the eigenvalues satisfy the inequality $\lambda_{s} \geq m, s=1,2, \ldots$, and there is a single eigenvalue equal to $m$ corresponding to eigenfunction

$$
\left(\sum_{E_{j} \in \mathfrak{E}} d_{j} \int_{0}^{l_{j}} c_{j}(x) d x\right)^{-1}(1,1, \ldots, 1, \ldots) .
$$

Theorem 2. Eigenfunctions $u_{1}(x), u_{2}(x), \ldots$ of problem $(2)-(4)$ form an orthonormal basis in $L_{2}(G)$, i.e. any function $f \in L_{2}(G)$ is decomposed in a Fourier series

$$
f=\sum_{s=1}^{\infty} f_{s} u_{s}, f_{s}=\left\langle f, u_{s}\right\rangle
$$

converging in $L_{2}(G)$. For a function $f \in \mathfrak{U}$ series $(11)$ converges in $\mathfrak{U}$ and

$$
\sum_{s=1}^{\infty}\left|\lambda_{s}\right|\left|f_{s}\right|^{2} \leq C|| f \|_{\mathfrak{U}}^{2}
$$

holds. Here the constant $C$ is independent of $f$.

\section{Examples of finding of eigenfunctions and eigenvalues of the Sturm - Liouville problem}

Example 1. Let graph $G$ consist of one direct edge of length $l$ connecting two vertices (Fig. 1).

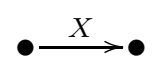

Fig. 1. Graph $G_{1}$

Let's find eigenfunctions and eigenvalues of the Sturm - Liouville problem for this graph. It is needed to find nontrivial solutions $X(x)$ (eigenfunctions) of the problem

$$
\left\{\begin{array}{l}
X^{\prime \prime}+\lambda X=0, \\
X^{\prime}(0)=X^{\prime}(l)=0
\end{array}\right.
$$

and eigenvalues $\lambda$.

Solving this problem we find eigenvalues:

$$
\lambda_{k}=\left(\frac{\pi k}{l}\right)^{2}
$$

and eigenfunctions:

$$
X^{k}=\sqrt{\frac{2}{l}} \cos \frac{\pi k}{l} x, k=1,2, \ldots
$$

Example 2. Let graph $G$ consist of two edges of lengths $l_{1}, l_{2}$ correspondingly, connecting three vertices (Fig. 2). 


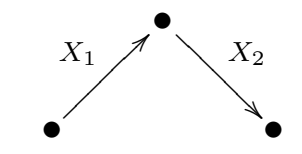

Fig. 2. Graph $G_{2}$

Let's find eigenfunctions and eigenvalues of the Sturm - Liouville problem for this graph. It is needed to find nontrivial solutions $X(x)=\left(X_{1}(x), X_{2}(x)\right)$ (eigenfunctions) of the problem:

$$
\left\{\begin{array}{l}
X_{1}^{\prime \prime}+\lambda X_{1}=0 \\
X_{2}^{\prime \prime}+\lambda X_{2}=0 \\
X_{1}\left(l_{1}\right)=X_{2}(0) \\
X_{1}^{\prime}\left(l_{1}\right)-X_{2}^{\prime}(0)=0 \\
X_{1}^{\prime}(0)=0 \\
X_{2}^{\prime}\left(l_{2}\right)=0
\end{array}\right.
$$

Solving this problem, we find eigenvalues:

$$
\lambda_{k}=\left(\frac{\pi k}{l_{1}+l_{2}}\right)^{2}
$$

and eigenfunctions:

$$
\begin{gathered}
X_{1}^{k}=\sqrt{\frac{2}{l_{1}+l_{2}}} \cos \frac{\pi k}{l_{1}+l_{2}} x, k=0,1, \ldots \\
X_{2}^{k}=\sqrt{\frac{2}{l_{1}+l_{2}}} \cos \frac{\pi k}{l_{1}+l_{2}}\left(x+l_{1}\right), k=0,1, \ldots
\end{gathered}
$$

Example 3. Let graph $G$ consist of three edges of lengths $l_{1}, l_{2}, l_{3}$ correspondingly, connecting four vertices (Fig. 3).

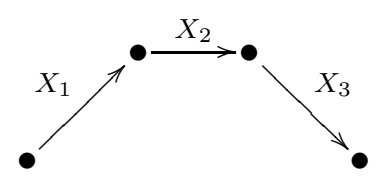

Fig. 3. Graph $G_{3}$

Let's find eigenfunctions and eigenvalues of the Sturm - Liouville problem for this graph. It is needed to find nontrivial solutions $X(x)=\left(X_{1}(x), X_{2}(x), X_{3}(x)\right)$ (eigenfunctions) of 
the problem:

$$
\left\{\begin{array}{l}
X_{1}^{\prime \prime}+\lambda X_{1}=0 \\
X_{2}^{\prime \prime}+\lambda X_{2}=0 \\
X_{3}^{\prime \prime}+\lambda X_{3}=0 \\
X_{1}^{\prime}(0)=0 \\
X_{1}^{\prime}\left(l_{1}\right)-X_{2}^{\prime}(0)=0 \\
X_{2}^{\prime}\left(l_{2}\right)-X_{3}^{\prime}(0)=0 \\
X_{3}^{\prime}\left(l_{3}\right)=0 \\
X_{1}\left(l_{1}\right)=X_{2}(0) \\
X_{2}\left(l_{2}\right)=X_{3}(0) .
\end{array}\right.
$$

Solving this problem, we find eigenvalues:

$$
\lambda_{k}=\left(\frac{\pi k}{l_{1}+l_{2}+l_{3}}\right)^{2},
$$

and eigenfunctions:

$$
\begin{gathered}
X_{1}^{k}=\sqrt{\frac{2}{l_{1}+l_{2}+l_{3}}} \cos \frac{\pi k}{l_{1}+l_{2}+l_{3}} x \\
X_{2}^{k}=\sqrt{\frac{2}{l_{1}+l_{2}+l_{3}}} \cos \frac{\pi k}{l_{1}+l_{2}+l_{3}}\left(x+l_{1}\right), \\
X_{3}^{k}=\sqrt{\frac{2}{l_{1}+l_{2}+l_{3}}} \cos \frac{\pi k}{l_{1}+l_{2}+l_{3}}\left(x+l_{1}+l_{2}\right), k=0,1, \ldots
\end{gathered}
$$

Example 4. Let graph $G$ consist of two edges of lengths $l_{1}, l_{2}$ correspondingly, connecting three vertices (Fig. 4).

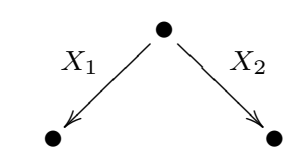

Fig. 4. Graph $G_{4}$

Let's find eigenfunctions and eigenvalues of the Sturm - Liouville problem for this graph. It is needed to find nontrivial solutions $X(x)=\left(X_{1}(x), X_{2}(x)\right)$ (eigenfunctions) of the problem:

$$
\left\{\begin{array}{l}
X_{1}^{\prime \prime}+\lambda X_{1}=0, \\
X_{2}^{\prime \prime}+\lambda X_{2}=0, \\
X_{1}(0)=X_{2}(0), \\
X_{1}^{\prime}\left(l_{1}\right)=0, \\
X_{2}^{\prime}\left(l_{2}\right)=0, \\
X_{1}^{\prime}(0)+X_{2}^{\prime}(0)=0 .
\end{array}\right.
$$

Solving this problem, we find eigenvalues:

$$
\lambda_{k}=\left(\frac{\pi k}{l_{1}+l_{2}}\right)^{2}
$$


and eigenfunctions:

1) If $\sin \frac{\pi k l_{1}}{l_{1}+l_{2}} \neq 0$,

$$
\begin{aligned}
& X_{1}^{k}=C_{2} \operatorname{ctg} \frac{\pi k l_{1}}{l_{1}+l_{2}} \cos \frac{\pi k x}{l_{1}+l_{2}}+C_{2} \sin \frac{\pi k x}{l_{1}+l_{2}}, k=0,1, \ldots \\
& X_{2}^{k}=C_{2} \operatorname{ctg} \frac{\pi k l_{1}}{l_{1}+l_{2}} \cos \frac{\pi k x}{l_{1}+l_{2}}-C_{2} \sin \frac{\pi k x}{l_{1}+l_{2}}, k=0,1, \ldots
\end{aligned}
$$

2) If $\sin \frac{\pi k l_{1}}{l_{1}+l_{2}}=0$,

$$
\begin{aligned}
& X_{1}^{k}=C_{1} \cos \frac{\pi k}{l_{1}+l_{2}} x, k=0,1, \ldots \\
& X_{2}^{k}=C_{1} \cos \frac{\pi k}{l_{1}+l_{2}} x, k=0,1, \ldots
\end{aligned}
$$

Example 5. Let graph $G$ consist of two edges of lengths $l_{1}, l_{2}$ correspondingly, connecting three vertices (Fig. 5).

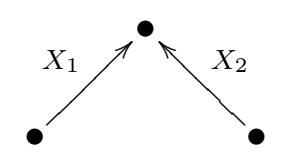

Fig. 5. Graph $G_{5}$

Let's find eigenfunctions and eigenvalues of the Sturm - Liouville problem for this graph. It is needed to find nontrivial solutions $X(x)=\left(X_{1}(x), X_{2}(x)\right)$ (eigenfunctions) of the problem:

$$
\left\{\begin{array}{l}
X_{1}^{\prime \prime}+\lambda X_{1}=0 \\
X_{2}^{\prime \prime}+\lambda X_{2}=0 \\
X_{1}^{\prime}(0)=0 \\
X_{2}^{\prime}(0)=0 \\
X_{1}^{\prime}\left(l_{1}\right)+X_{2}^{\prime}\left(l_{2}\right)=0 \\
X_{1}\left(l_{1}\right)=X_{2}\left(l_{2}\right) .
\end{array}\right.
$$

Solving this problem, we find eigenvalues: $\lambda_{k}=\left(\frac{\pi k}{l_{1}+l_{2}}\right)^{2}$.

and eigenfunctions:

1) If $\sin \frac{\pi k l_{1}}{l_{1}+l_{2}} \neq 0$, then

$$
X_{1}^{k}=-C_{3} \frac{\sin \frac{\pi k}{l_{1}+l_{2}} l_{2}}{\sin \frac{\pi k}{l_{1}+l_{2}} l_{1}} \cos \frac{\pi k}{l_{1}+l_{2}} x, X_{2}^{k}=C_{3} \cos \frac{\pi k}{l_{1}+l_{2}} x, k=0,1, \ldots
$$

2) If $\sin \frac{\pi k l_{1}}{l_{1}+l_{2}}=0$, then

$$
X_{1}^{k}=C_{3} \cos \frac{\pi k}{l_{1}+l_{2}} l_{2} \cos \frac{\pi k}{l_{1}+l_{2}} x, X_{2}^{k}=C_{3} \cos \frac{\pi k}{l_{1}+l_{2}} x, k=0,1, \ldots
$$




\section{Fourier Method for a Boussinesq - Löve mathematical model on a geometric graph}

It is needed to find not identically equal to zero solution of equation

$$
\lambda u_{t t}-u_{x x t t}=\alpha u_{x x}
$$

on a geometric graph $G$ satisfying boundary conditions

$$
\begin{gathered}
\sum_{E_{j} \in E^{a}\left(V_{i}\right)} d_{j} u_{j x}(0, t)-\sum_{E_{m} \in E^{\omega}\left(V_{i}\right)} d_{m} u_{m x}\left(l_{m}, t\right)=0, \\
u_{j}(0, t)=u_{k}(0, t)=u_{m}\left(l_{m}, t\right)=u_{n}\left(l_{n}, t\right)
\end{gathered}
$$

and initial conditions

$$
\left\{\begin{array}{l}
u(x, 0)=\varphi(x), \\
u_{t}(x, 0)=\psi(x) .
\end{array}\right.
$$

We will search the solution of (14)-(17) in the form

$$
u(x, t)=\sum_{k=1}^{\infty} T^{k}(t) X^{k}(x),
$$

where $X^{k}(x)$ are orthonormal eigenfunctions of the operator $-\frac{\partial^{2}}{\partial x^{2}}$, i.e. satisfying equation $\left(X^{k}\right) "+\lambda_{k} X^{k}=0$, and conditions (15), (16) and $T^{k}(t)$ are functions of the variable $t$.

Equation (14) is linear and homogeneous, so the sum of particular solutions is also a solution of this equation. Substituting the assumed form of solution (18) into equation (14), we obtain

$$
\begin{gathered}
\lambda \sum_{k=1}^{\infty}\left(T^{k}\right)^{\prime \prime}(t) X^{k}(x)+\sum_{k=1}^{\infty}\left(T^{k}\right)^{\prime \prime}(t) \lambda_{k} X^{k}(x)=-\alpha \sum_{k=1}^{\infty} T^{k}(t) \lambda_{k} X^{k}(x) \\
\lambda \sum_{k=1}^{\infty}\left(T^{k}\right)^{\prime \prime}(t) X^{k}(x)+\sum_{k=1}^{\infty}\left(T^{k}\right)^{\prime \prime}(t) \lambda_{k} X^{k}(x)+\alpha \sum_{k=1}^{\infty} T^{k}(t) \lambda_{k} X^{k}(x)=0 \\
\sum_{k=1}^{\infty} X^{k}(x)\left(\lambda\left(T^{k}\right)^{\prime \prime}(t)+\left(T^{k}\right)^{\prime \prime}(t) \lambda_{k}+\alpha T^{k}(t) \lambda_{k}\right)=0
\end{gathered}
$$

Multiplying the resulting equation by $X^{s}(x), s=0,1, \ldots$ in $L_{2}(G)$ we get

$$
\lambda\left(T^{k}\right)^{\prime \prime}(t)+\left(T^{k}\right)^{\prime \prime}(t) \lambda_{k}+\alpha T^{k}(t) \lambda_{k}=0
$$

or

$$
\left(\lambda+\lambda_{k}\right)\left(T^{k}\right)^{\prime \prime}(t)+\alpha T^{k}(t) \lambda_{k}=0 .
$$

The characteristic equation for this differential equation has the form

$$
\left(\lambda+\lambda_{k}\right) \mu_{k}^{2}+\alpha \lambda_{k}=0
$$


where

$$
\mu_{k}^{2}=\frac{-\alpha \lambda_{k}}{\lambda+\lambda_{k}}
$$

Consider the cases

1) If $\lambda_{k}=-\lambda$ then equation (19) has no nontrivial solutions. Therefore

$$
T^{k}=0 .
$$

2) If $\lambda_{k}>-\lambda$ then

$$
T^{k}=A_{k} \cos \sqrt{\frac{\alpha \lambda_{k}}{\lambda+\lambda_{k}}} t+B_{k} \sin \left(\sqrt{\frac{\alpha \lambda_{k}}{\lambda+\lambda_{k}}} t\right.
$$

3) If $\lambda_{k}<-\lambda$ then

$$
T^{k}=A_{k} e^{\sqrt{\frac{-\alpha \lambda_{k}}{\lambda+\lambda_{k}}} t}+B_{k} e^{-\sqrt{\frac{-\alpha \lambda_{k}}{\lambda+\lambda_{k}}} t}
$$

Combining cases 1) and 2) and returning to problem (14) - (16), we conclude that the functions

$$
u_{k}(x, t)= \begin{cases}\left(A_{k} e^{\sqrt{\frac{-\alpha \lambda_{k}}{\lambda+\lambda_{k}}} t}+B_{k} e^{-\sqrt{\frac{-\alpha \lambda_{k}}{\lambda+\lambda_{k}}} t}\right) X^{k}, & \lambda_{k}<-\lambda \\ \left(A_{k} \cos \sqrt{\frac{\alpha \lambda_{k}}{\lambda+\lambda_{k}}} t+B_{k} \sin \left(\sqrt{\frac{\alpha \lambda_{k}}{\lambda+\lambda_{k}}}(t) X^{k},\right.\right. & \lambda_{k}>-\lambda\end{cases}
$$

are particular solutions of equation (14) satisfying boundary conditions (15), (16). These solutions satisfy initial conditions (17) of the original problem only for particular cases of initial functions $\varphi(x)$ and $\psi(x)$.

Due to the linearity and homogeneity of equation (14) the sum of particular solutions

$$
\begin{gathered}
u(x, t)=\sum_{k \lambda_{k}<-\lambda}\left(A_{k} e^{\sqrt{\frac{-\alpha \lambda_{k}}{\lambda+\lambda_{k}}} t}+B_{k} e^{\left.-\sqrt{\frac{-\alpha \lambda_{k}}{\lambda+\lambda_{k}}}\right) X^{k}+}\right. \\
+\sum_{k \lambda_{k}>-\lambda}\left(A_{k} \cos \sqrt{\frac{\alpha \lambda_{k}}{\lambda+\lambda_{k}}} t+B_{k} \sin \left(\sqrt{\frac{\alpha \lambda_{k}}{\lambda+\lambda_{k}}}(t) X^{k}\right.\right.
\end{gathered}
$$

also satisfies this equation and boundary conditions (15), (16). Here $A_{k}, B_{k}$ are arbitrary constants.

Initial conditions allow to define constants $A_{k}, B_{k}$. We require that function (20) satisfies conditions (17):

$$
\begin{gathered}
u(x, 0)=\sum_{k=1}^{\infty} u_{k}(x, 0)=\sum_{k \lambda_{k}<-\lambda}\left(A_{k}+B_{k}\right) X^{k}+\sum_{k \lambda_{k}>-\lambda} A_{k} X^{k}=\varphi(x), \\
u_{t}(x, 0)=\sum_{k=1}^{\infty} \frac{\partial u_{k}}{\partial t}(x, 0)=\sum_{k \lambda_{k}<-\lambda}\left(A_{k} \sqrt{\frac{-\alpha \lambda_{k}}{\lambda+\lambda_{k}}}-B_{k} \sqrt{\frac{-\alpha \lambda_{k}}{\lambda+\lambda_{k}}}\right) X^{k}+ \\
+\sum_{k \lambda_{k}>-\lambda}\left(B_{k} \sqrt{\frac{\alpha \lambda_{k}}{\lambda+\lambda_{k}}}\right) X^{k}=\psi(x) .
\end{gathered}
$$


On the other hand decompose the functions $\varphi(x)$ and $\psi(x)$ as a series in eigenfunctions $X^{k}(x)$ :

$$
\begin{aligned}
& \varphi(x)=\sum_{k=1}^{\infty} \varphi_{k} X^{k}, \text { where } \varphi_{k}=<\varphi, X^{k}>_{L_{2}(G)}, \\
& \psi(x)=\sum_{k=1}^{\infty} \psi_{k} X^{k}, \quad \text { where } \psi_{k}=<\psi, X^{k}>_{L_{2}(G)} .
\end{aligned}
$$

Consider two cases:

1) If $\lambda_{k}<-\lambda$ then

$$
\left\{\begin{array}{l}
A_{k}+B_{k}=\varphi_{k} \\
A_{k} \sqrt{\frac{-\alpha \lambda_{k}}{\lambda+\lambda_{k}}}-B_{k} \sqrt{\frac{-\alpha \lambda_{k}}{\lambda+\lambda_{k}}}=\psi_{k}
\end{array}\right.
$$

where

$$
\left\{\begin{array}{l}
B_{k}=\frac{\varphi_{k}}{2}-\frac{\psi_{k}}{2 \sqrt{\frac{-\alpha \lambda_{k}}{\lambda+\lambda_{k}}}}, \\
A_{k}=\frac{\varphi_{k}}{2}+\frac{\psi_{k}}{2 \sqrt{\frac{-\alpha \lambda_{k}}{\lambda+\lambda_{k}}}} .
\end{array}\right.
$$

2) If $\lambda_{k}>-\lambda$ then

$$
\left\{\begin{array}{l}
A_{k}=\varphi_{k} \\
B_{k}=\frac{\psi_{k}}{\sqrt{\frac{\alpha \lambda_{k}}{\lambda+\lambda_{k}}}}
\end{array}\right.
$$

\section{Justification of the Fourier method for a Boussinesq - Löve mathematical model on a geometric graph}

First of all we need to check the continuity of the function

$$
\begin{gathered}
u(x, t)=\sum_{k \lambda_{k}<-\lambda}\left(A_{k} e^{\sqrt{\frac{-\alpha \lambda_{k}}{\lambda+\lambda_{k}}} t}+B_{k} e^{\left.-\sqrt{\frac{-\alpha \lambda_{k}}{\lambda+\lambda_{k}}}\right) X^{k}+}\right. \\
+\sum_{k \lambda_{k}>-\lambda}\left(A_{k} \cos \sqrt{\frac{\alpha \lambda_{k}}{\lambda+\lambda_{k}}} t+B_{k} \sin \left(\sqrt{\frac{\alpha \lambda_{k}}{\lambda+\lambda_{k}}}(t) X^{k} .\right.\right.
\end{gathered}
$$

Since the first sum has a finite number of summands, it is sufficient to prove uniform convergence of the series

$$
v(x, t)=\sum_{k \lambda_{k}>-\lambda}\left(A_{k} \cos \sqrt{\frac{\alpha \lambda_{k}}{\lambda+\lambda_{k}}} t+B_{k} \sin \left(\sqrt{\frac{\alpha \lambda_{k}}{\lambda+\lambda_{k}}}(t) X^{k} .\right.\right.
$$

It follows that $u(x, t)$ is continuously adjacent to its initial and boundary conditions. As the terms of series (22) are continuous functions, uniform convergence of series is sufficient for its continuity. Using the inequality

$$
\left|v_{k}(x, t)\right| \leq\left|A_{k}\right|+\left|B_{k}\right|,
$$


we conclude that the series

$$
\sum_{k=1}^{\infty}\left(\left|\varphi_{k}\right|+\frac{1}{\sqrt{\frac{\alpha \lambda_{k}}{\lambda+\lambda_{k}}}}\left|\psi_{k}\right|\right)
$$

is a majorant for (22). If a majorant series (23) converges, then (22) converges uniformly, i.e., the function $u(x, t)$ is continuous.

To confirm that $u_{t}(x, t)$ is continuously adjacent to its initial values, we need to prove the continuity of this function, i.e. to prove the uniform convergence of series

$$
\begin{gathered}
v_{t}(x, t) \sim \sum_{k=1}^{\infty} \frac{\partial v_{k}}{\partial t}=\sum_{k=1}^{\infty} \sqrt{\frac{\alpha \lambda_{k}}{\lambda+\lambda_{k}}}\left(-\varphi_{k} \sin \left(\sqrt{\frac{\alpha \lambda_{k}}{\lambda+\lambda_{k}}} t+\right.\right. \\
+\frac{\psi_{k}}{\sqrt{\frac{\alpha \lambda_{k}}{\lambda+\lambda_{k}}}} \cos \sqrt{\frac{\alpha \lambda_{k}}{\lambda+\lambda_{k}}}(t) X^{k}
\end{gathered}
$$

or the convergence of a majorant series

$$
\sum_{k=1}^{\infty} \sqrt{\frac{\alpha \lambda_{k}}{\lambda+\lambda_{k}}}\left(\left|\varphi_{k}\right|+\frac{1}{\sqrt{\frac{\alpha \lambda_{k}}{\lambda+\lambda_{k}}}}\left|\psi_{k}\right|\right) .
$$

Finally, to make sure that the function $v(x, t)$ satisfies the equation, i.e. apply the generalized principle of superposition, it is sufficient to prove the possibility of double memberwise differentiation of the series for $v(x, t)$, i.e. to show the uniform convergence of series

$$
\begin{aligned}
v_{t t}(x, t) & \sim \sum_{k=1}^{\infty} \frac{\partial^{2} v_{k}}{\partial t^{2}}, \\
v_{x x}(x, t) & \sim \sum_{k=1}^{\infty} \frac{\partial^{2} v_{k}}{\partial x^{2}}, \\
v_{x x t t}(x, t) & \sim \sum_{k=1}^{\infty} \frac{\partial^{4} v_{k}}{\partial x^{2} \partial t^{2}} .
\end{aligned}
$$

Investigate the uniform convergence of series $(26)$ - (28) for one-edged graph from example 1 . In this case

$$
\begin{aligned}
& v(x, t)=\sum_{k=1}^{\infty}\left(\varphi_{k} \cos \sqrt{\frac{\alpha \lambda_{k}}{\lambda+\lambda_{k}}} t+\frac{\psi_{k}}{\sqrt{\frac{\alpha \lambda_{k}}{\lambda+\lambda_{k}}}} \sin \left(\sqrt{\frac{\alpha \lambda_{k}}{\lambda+\lambda_{k}}}(t) \sqrt{\frac{2}{l}} \cos \frac{\pi k}{l} x,\right.\right. \\
& v_{t t}(x, t) \sim \sqrt{\frac{2}{l}} \sum_{k=1}^{\infty} \frac{-\alpha \lambda_{k}}{\lambda+\lambda_{k}}\left(\varphi_{k} \cos \sqrt{\frac{\alpha \lambda_{k}}{\lambda+\lambda_{k}}} t+\frac{\psi_{k}}{\sqrt{\frac{\alpha \lambda_{k}}{\lambda+\lambda_{k}}}} \sin \left(\sqrt{\frac{\alpha \lambda_{k}}{\lambda+\lambda_{k}}}(t) \cos \frac{\pi k}{l} x\right.\right. \\
& v_{x x}(x, t) \sim \sqrt{\frac{2}{l}} \sum_{k=1}^{\infty}-\frac{\pi^{2} k^{2}}{l^{2}}\left(\varphi_{k} \cos \sqrt{\frac{\alpha \lambda_{k}}{\lambda+\lambda_{k}}} t+\frac{\psi_{k}}{\sqrt{\frac{\alpha \lambda_{k}}{\lambda+\lambda_{k}}}} \sin \left(\sqrt{\frac{\alpha \lambda_{k}}{\lambda+\lambda_{k}}}(t) \cos \frac{\pi k}{l} x,\right.\right.
\end{aligned}
$$




$$
\begin{aligned}
v_{x x t t}(x, t) & \sim \sqrt{\frac{2}{l}} \sum_{k=1}^{\infty} \frac{\pi^{2} k^{2} \alpha \lambda_{k}}{l^{2}\left(\lambda+\lambda_{k}\right)}\left(\varphi_{k} \cos \sqrt{\frac{\alpha \lambda_{k}}{\lambda+\lambda_{k}}} t+\right. \\
+ & \frac{\psi_{k}}{\sqrt{\frac{\alpha \lambda_{k}}{\lambda+\lambda_{k}}}} \sin \left(\sqrt{\frac{\alpha \lambda_{k}}{\lambda+\lambda_{k}}}(t) \cos \frac{\pi k}{l} x,\right.
\end{aligned}
$$

which is with accuracy to the multipliers of proportionality corresponds to the majorant series

$$
\sum_{k=1}^{\infty} k^{2}\left(\left|\varphi_{k}\right|+\left|\psi_{k}\right|\right) .
$$

Therefore the problem is reduced to the proof of convergence of the series

$$
\begin{aligned}
& \sum_{k=1}^{\infty} k^{n}\left|\varphi_{k}\right|, \quad(n=0,1,2), \\
& \sum_{k=1}^{\infty} k^{n}\left|\psi_{k}\right|, \quad(n=0,1,2) .
\end{aligned}
$$

For this purpose, we use the known properties of Fourier series. If a periodic with period $2 l$ function $F(x)$ has $k$ continuous derivatives, and its $(k+1)$-th derivative is piecewise continuous, then the series

$$
\sum_{k=1}^{\infty}\left(k^{n}\left|A_{k}\right|+\left|B_{k}\right|\right),
$$

where $A_{k}$ and $B_{k}$ are a Fourier coefficients, converges. For decomposition in the series by $\cos \frac{\pi k}{l} x$ of a function $f(x)$ defined only in the interval $(0, l)$, it is necessary that the preceding requirements are met for the function $F(x)$, resulting in an even extension of $f(x)$.

The continuity of function $F(x)$ at $x=0, x=l$ is obtained automatically. For the continuity of $F^{\prime}(x)$ it is required that $f^{\prime}(0)=0$, since otherwise $F^{\prime}(x)$ will break at the point $x=0$. Similarly require that $f^{\prime}(l)=0$. In general, for the continuity of odd derivatives of extension of function $f$ it is necessary to require that

$$
f^{(2 n+1)}(0)=f^{(2 n+1)}(l)=0,(n=0,1,2, \ldots) .
$$

Continuity of even derivatives takes place without any additional requirements. So, for convergence of series

$$
\sum_{n=1}^{\infty} k^{n}\left|\varphi_{k}\right|, \quad(n=0,1,2)
$$

it is sufficient to require that the initial deviation $\varphi(x)$ satisfies the following requirements: Derivatives of function $\varphi(x)$ to the second order inclusively are continuous, the third order derivative of the function $\varphi(x)$ is piecewise continuous and, in addition,

$$
\varphi^{\prime}(0)=\varphi^{\prime}(l)=0 .
$$

For the convergence of series

$$
\sum_{k=1}^{\infty} k^{n}\left|\psi_{k}\right|, \quad(n=0,1,2)
$$


it is sufficient to impose the following requirements:

derivatives of function $\psi(x)$ to the second order inclusively are continuous, the third order derivative of the function $\psi(x)$ is piecewise continuous and, in addition,

$$
\psi^{\prime}(0)=\psi^{\prime}(l)=0
$$

Theorem 3. Let the derivatives of functions $\varphi(x), \psi(x)$ to the second order inclusively, be continuous, the third order derivatives of functions $\varphi(x), \psi(x)$ be piecewise continuous and, in addition, (33), (34) hold. Then there exists a unique solution of problem (14)(17) on one-edged graph $G_{1}$ which can be represented as:

$$
\begin{gathered}
u(x, t)=\sum_{k \lambda_{k}>-\lambda}\left[\varphi_{k} \cos \sqrt{\frac{\alpha \lambda_{k}}{\lambda+\lambda_{k}}} t+\frac{\psi_{k}}{\sqrt{\frac{\alpha \lambda_{k}}{\lambda+\lambda_{k}}}} \sin \left(\sqrt{\frac{\alpha \lambda_{k}}{\lambda+\lambda_{k}}} t\right] X_{1}^{k}+\right. \\
+\sum_{k \lambda_{k}<-\lambda}\left[\left(\frac{\varphi_{k}}{2}+\frac{\psi_{k}}{2 \sqrt{\frac{-\alpha \lambda_{k}}{\lambda+\lambda_{k}}}}\right) e^{\sqrt{\frac{-\alpha \lambda_{k}}{\lambda+\lambda_{k}}}}+\left(\frac{\varphi_{k}}{2}-\frac{\psi_{k}}{2 \sqrt{\frac{-\alpha \lambda_{k}}{\lambda+\lambda_{k}}}}\right) e^{-\sqrt{\frac{-\alpha \lambda_{k}}{\lambda+\lambda_{k}}} t}\right] X_{1}^{k},
\end{gathered}
$$

where $X_{1}^{k}=\sqrt{\frac{2}{l}} \cos \frac{\pi k}{l} x, \lambda_{k}=\left(\frac{\pi k}{l}\right)^{2}$.

Similarly, the following theorems on existence and uniqueness of solution for a graphs $G_{2}$ and $G_{3}$ can be proved:

Theorem 4. Let the derivatives of functions $\varphi(x), \psi(x)$ to the second order inclusively be continuous, the third order derivatives of functions $\varphi(x), \psi(x)$ be piecewise continuous and, in addition,

$$
\begin{aligned}
& \varphi_{1}\left(l_{1}\right)=\varphi_{2}(0)=0, \varphi_{1}^{\prime}\left(l_{1}\right)=\varphi_{1}^{\prime}(0)=0 \\
& \varphi_{2}^{\prime}\left(l_{2}\right)=\varphi_{2}^{\prime}(0)=0, \varphi_{1}^{\prime \prime}\left(l_{1}\right)=\varphi_{2}^{\prime \prime}(0)=0 \\
& \psi_{1}\left(l_{1}\right)=\psi_{2}(0)=0, \psi_{1}^{\prime}\left(l_{1}\right)=\psi_{1}^{\prime}(0)=0 \\
& \psi_{2}^{\prime}\left(l_{2}\right)=\psi_{2}^{\prime}(0)=0, \psi_{1}^{\prime \prime}\left(l_{1}\right)=\psi_{2}^{\prime \prime}(0)=0 .
\end{aligned}
$$

Then, there exists a unique solution of problem (14) - (17) on graph $G_{2}$ which can be represented as:

$$
\begin{gathered}
u_{1}(x, t)=\sum_{k \lambda_{k}>-\lambda}\left[\varphi_{k} \cos \sqrt{\frac{\alpha \lambda_{k}}{\lambda+\lambda_{k}}} t+\frac{\psi_{k}}{\sqrt{\frac{\alpha \lambda_{k}}{\lambda+\lambda_{k}}}} \sin \left(\sqrt{\frac{\alpha \lambda_{k}}{\lambda+\lambda_{k}}} t\right] X_{1}^{k}+\right. \\
+\sum_{k: \lambda_{k}<-\lambda}\left[\left(\frac{\varphi_{k}}{2}+\frac{\psi_{k}}{2 \sqrt{\frac{-\alpha \lambda_{k}}{\lambda+\lambda_{k}}}}\right) e^{\sqrt{\frac{-\alpha \lambda_{k}}{\lambda+\lambda_{k}}} t}+\left(\frac{\varphi_{k}}{2}-\frac{\psi_{k}}{2 \sqrt{\frac{-\alpha \lambda_{k}}{\lambda+\lambda_{k}}}}\right) e^{-\sqrt{\frac{-\alpha \lambda_{k}}{\lambda+\lambda_{k}}} t}\right] X_{1}^{k}, \\
u_{2}(x, t)=\sum_{k \lambda_{k}>-\lambda}\left[\varphi_{k} \cos \sqrt{\frac{\alpha \lambda_{k}}{\lambda+\lambda_{k}}} t+\frac{\psi_{k}}{\sqrt{\frac{\alpha \lambda_{k}}{\lambda+\lambda_{k}}}} \sin \left(\sqrt{\frac{\alpha \lambda_{k}}{\lambda+\lambda_{k}}} t\right] X_{2}^{k}+\right.
\end{gathered}
$$




$$
+\sum_{k \lambda_{k}<-\lambda}\left[\left(\frac{\varphi_{k}}{2}+\frac{\psi_{k}}{2 \sqrt{\frac{-\alpha \lambda_{k}}{\lambda+\lambda_{k}}}}\right) e^{\sqrt{\frac{-\alpha \lambda_{k}}{\lambda+\lambda_{k}}} t}+\left(\frac{\varphi_{k}}{2}-\frac{\psi_{k}}{2 \sqrt{\frac{-\alpha \lambda_{k}}{\lambda+\lambda_{k}}}}\right) e^{-\sqrt{\frac{-\alpha \lambda_{k}}{\lambda+\lambda_{k}}} t}\right] X_{2}^{k}
$$

where

$$
\begin{gathered}
X_{1}^{k}=\sqrt{\frac{2}{l_{1}+l_{2}}} \cos \frac{\pi k}{l_{1}+l_{2}} x, \\
X_{2}^{k}=\sqrt{\frac{2}{l_{1}+l_{2}}} \cos \frac{\pi k}{l_{1}+l_{2}}\left(x+l_{1}\right), \\
\lambda_{k}=\left(\frac{\pi k}{l_{1}+l_{2}}\right)^{2} .
\end{gathered}
$$

Theorem 5. Let the derivatives of functions $\varphi(x) \psi(x)$ to the second order inclusively be continuous, the third order derivatives of functions $\varphi(x) \psi(x)$ be piecewise continuous and, in addition,

$$
\begin{gathered}
\varphi_{1}\left(l_{1}\right)=\varphi_{2}(0)=\varphi_{2}\left(l_{2}\right)=\varphi_{3}(0)=0, \\
\varphi_{1}^{\prime}\left(l_{1}\right)=\varphi_{1}^{\prime}(0)=0, \varphi_{2}^{\prime}\left(l_{2}\right)=\varphi_{2}^{\prime}(0)=0, \varphi_{3}^{\prime}\left(l_{3}\right)=\varphi_{2}^{\prime}(0)=0, \\
\varphi_{1}^{\prime \prime}\left(l_{1}\right)=\varphi_{2}^{\prime \prime}(0)=\varphi_{2}^{\prime \prime}\left(l_{2}\right)=\varphi_{3}^{\prime \prime}(0)=0, \\
\psi_{1}\left(l_{1}\right)=\psi_{2}(0)=\psi_{2}\left(l_{2}\right)=\psi_{3}(0)=0, \\
\psi_{1}^{\prime}\left(l_{1}\right)=\psi_{1}^{\prime}(0)=0, \psi_{2}^{\prime}\left(l_{2}\right)=\psi_{2}^{\prime}(0)=0, \psi_{3}^{\prime}\left(l_{3}\right)=\psi_{2}^{\prime}(0)=0, \\
\psi_{1}^{\prime \prime}\left(l_{1}\right)=\psi_{2}^{\prime \prime}(0)=\psi_{2}^{\prime \prime}\left(l_{2}\right)=\psi_{3}^{\prime \prime}(0)=0,
\end{gathered}
$$

Then, there exists a unique solution of problem (14) - (17) on graph $G_{3}$ which can be represented as:

$$
\begin{gathered}
u_{1}(x, t)=\sum_{k \lambda_{k}>-\lambda}\left[\varphi_{k} \cos \sqrt{\frac{\alpha \lambda_{k}}{\lambda+\lambda_{k}}} t+\frac{\psi_{k}}{\sqrt{\frac{\alpha \lambda_{k}}{\lambda+\lambda_{k}}}} \sin \left(\sqrt{\frac{\alpha \lambda_{k}}{\lambda+\lambda_{k}}} t\right] X_{1}^{k}+\right. \\
+\sum_{k \lambda_{k}<-\lambda}\left[\left(\frac{\varphi_{k}}{2}+\frac{\psi_{k}}{\left.2 \sqrt{\frac{-\alpha \lambda_{k}}{\lambda+\lambda_{k}}}\right)}\right) e^{\sqrt{\frac{-\alpha \lambda_{k}}{\lambda+\lambda_{k}}} t}+\left(\frac{\varphi_{k}}{2}-\frac{\psi_{k}}{2 \sqrt{\frac{-\alpha \lambda_{k}}{\lambda+\lambda_{k}}}}\right) e^{-\sqrt{\frac{-\alpha \lambda_{k}}{\lambda+\lambda_{k}}} t}\right] X_{1}^{k}, \\
u_{2}(x, t)=\sum_{k \lambda_{k}>-\lambda}\left[\varphi_{k} \cos \sqrt{\frac{\alpha \lambda_{k}}{\lambda+\lambda_{k}}} t+\frac{\psi_{k}}{\sqrt{\frac{\alpha \lambda_{k}}{\lambda+\lambda_{k}}}} \sin \left(\sqrt{\frac{\alpha \lambda_{k}}{\lambda+\lambda_{k}}} t\right] X_{2}^{k}+\right. \\
\sum_{k \lambda_{k}<-\lambda}\left[\left(\frac{\varphi_{k}}{2}+\frac{\psi_{k}}{2 \sqrt{\frac{-\alpha \lambda_{k}}{\lambda+\lambda_{k}}}}\right) e^{\sqrt{\frac{-\alpha \lambda_{k}}{\lambda+\lambda_{k}}} t}+\left(\frac{\varphi_{k}}{2}-\frac{\psi_{k}}{2 \sqrt{\frac{-\alpha \lambda_{k}}{\lambda+\lambda_{k}}}}\right) e^{-\sqrt{\frac{-\alpha \lambda_{k}}{\lambda+\lambda_{k}}} t}\right] X_{2}^{k}, \\
u_{3}(x, t)=\sum_{k \lambda_{k}>-\lambda}\left[\varphi_{k} \cos \sqrt{\frac{\alpha \lambda_{k}}{\lambda+\lambda_{k}}} t+\frac{\psi_{k}}{\sqrt{\frac{\alpha \lambda_{k}}{\lambda+\lambda_{k}}}} \sin \left(\sqrt{\frac{\alpha \lambda_{k}}{\lambda+\lambda_{k}}} t\right] X_{3}^{k}+\right.
\end{gathered}
$$




$$
+\sum_{k \lambda_{k}<-\lambda}\left[\left(\frac{\varphi_{k}}{2}+\frac{\psi_{k}}{2 \sqrt{\frac{-\alpha \lambda_{k}}{\lambda+\lambda_{k}}}}\right) e^{\sqrt{\frac{-\alpha \lambda_{k}}{\lambda+\lambda_{k}}} t}+\left(\frac{\varphi_{k}}{2}-\frac{\psi_{k}}{2 \sqrt{\frac{-\alpha \lambda_{k}}{\lambda+\lambda_{k}}}}\right) e^{-\sqrt{\frac{-\alpha \lambda_{k}}{\lambda+\lambda_{k}}} t}\right] X_{3}^{k},
$$

where

$$
\begin{gathered}
X_{1}^{k}=\sqrt{\frac{2}{l_{1}+l_{2}+l_{3}}} \cos \frac{\pi k}{l_{1}+l_{2}+l_{3}} x, \\
X_{2}^{k}=\sqrt{\frac{2}{l_{1}+l_{2}+l_{3}}} \cos \frac{\pi k}{l_{1}+l_{2}+l_{3}}\left(x+l_{1}\right), \\
X_{3}^{k}=\sqrt{\frac{2}{l_{1}+l_{2}+l_{3}}} \cos \frac{\pi k}{l_{1}+l_{2}+l_{3}}\left(x+l_{1}+l_{2}\right), \\
\lambda_{k}=\left(\frac{\pi k}{l_{1}+l_{2}+l_{3}}\right)^{2} .
\end{gathered}
$$

\section{References}

1. Löve A.E.H. A Tretise on the Mathematical Theory of Elasticity. Cambridge, Univ. Press, 1927.

2. Zamyshlyaeva A.A. [Phase Spaces of Some Class of Linear Second Order Sobolev Type Equations]. Vychislitel'nyye Tehnologii - Computational Technologies, 2003, vol. 8, no. 4, pp. 45-54. (in Russian)

3. Zamyshlyaeva A.A., Yuzeeva A.V. The Initial-finish Value Problem for the Boussinesq - Löve Equation. Bulletin of the South Ural State University. Series "Mathematical Modelling, Programming 65 Computer Software", 2010, vol. 5, no. 16(192), pp. 23-31. (in Russian)

4. Zamyshlyaeva A.A., Tsyplenkova O.N. The Optimal Control over Solutions of the Initial-finish Value Problem for the Boussinesque - Löve Equation. Bulletin of the South Ural State University. Series "Mathematical Modelling, Programming \&6 Computer Software", 2012, vol. 11, no. 5(264), pp. 13-24. (in Russian)

5. Zamyshlyaeva A.A., Tsyplenkova O.N. Optimal Control of Solutions of the Showalter - Sidorov - Dirichlet Problem for the Boussinesq - Löve Equation. Differential Equations, 2013, vol. 49, no. 11, pp. 1356-1365.

6. Zamyshlyaeva A.A., Bychkov E.V. The Phase Space of the Modified Boussinesq Equation. Bulletin of the South Ural State University. Series "Mathematical Modelling, Programming \& Computer Software", 2012, vol. 12, no. 18(277), pp. 13-19. (in Russian)

7. Zamyshlyaeva A.A. On Algorithm of Numerical Modelling of the Boussinesq - Löve Waves. Bulletin of the South Ural State University. Series "Computer Technologies, Automatic Control \& Radioelectronics", 2013, vol. 13, no. 4, pp. 24-29. (in Russian)

8. Pokorny Y.V., Penkin O.M., Pryadiev V.L., Borovskih A.V., Lazarev K.P., Shabrov S.A. Differential Equations on Geometric Graphs. Moscow, Fizmatlit Publ., 2005. (in Russian) 
9. Sviridyuk G.A., Shemetova V.V. The Phase Space of One Nonclssical Model. Izv. Vyssh. Uchebn. Zaved. Mat., 2005, no. 11, pp. 47-52. (in Russian)

10. Sviridyuk G.A., Bayazitova A.A. On Direct and Inverse Problems for the Hoff Equations on Graph. Vestn. Samar. Gos. Tekhn. Univ. Ser. Fiz.-Mat. Nauki, 2009, vol. 18, no. 1, pp. 6-17. (in Russian)

11. Bayazitova A.A. The Sturm - Liouville Problem on Geometric Graph. Bulletin of the South Ural State University. Series "Mathematical Modelling, Programming 8 Computer Software", 2010, vol. 5, no. 16(192), pp. 4-10. (in Russian)

Alyona A. Zamyshlyaeva, Doctor of Physico-Mathematical Sciences, Docent, Department of Equation of Mathematical Physics, South Ural State University, Chelyabinsk, Russian Federation, alzama@mail.ru.

Aleksandr V. Lut, student, Faculty of Mathematics, Mechanics and Computer Sciences, South Ural State University, Chelyabinsk, Russian Federation, lutti74@rambler.ru

Received April 25, 2015 